\section{Análisis crítico de un artículo: La suspensión de inhibidores de la bomba de protones induciría síntomas de reflujo}

\author{
ROBERTO CANDIA B. ${ }^{1,2}$
}

\section{Critically appraised article}

\begin{tabular}{c}
\hline Reimer C, Søndergaard B, Hilsted L,Bytzer P. \\
Proton-Pump Inhibitor Therapy Induces Acid-Related \\
Symptoms in Healthy Volunteers After Withdrawal of Therapy. \\
Gastroenterology 2009; 137: 80-7
\end{tabular}

Background \& Aims: Rebound acid hypersecretion (RAHS) has been demonstrated after 8 weeks of treatment with a proton-pump inhibitor (PPI). If RAHS induces acid-related symptoms, this might lead to PPI dependency and thus have important implications. Methods: A randomized, double-blind, placebo-controlled trial with 120 healthy volunteers was conducted. Participants were randomized to 12 weeks of placebo or 8 weeks of esomeprazole $40 \mathrm{mg} / \mathrm{d}$ followed by 4 weeks with placebo. The Gastrointestinal Symptom Rating Scale (GSRS) was filled out weekly. A score of $>2$ on 1 of the questions regarding heartburn, acid regurgitation, or dyspepsia was defined as a clinically relevant acid-related symptom. Results: There were no significant differences between groups in GSRS scores at baseline. GSRS scores for acid-related symptoms were significantly higher in the PPI group at week 10 (1.4 \pm 1.4 vs $1.2 \pm 0.9 ; P=.023)$, week 11 (1.4 \pm 1.4 vs $1.2 \pm 0.9 ; P=.009)$, and week 12 $(1.3 \pm 1.2$ vs $1.0 \pm 0.3 ; P=.001)$. Forty-four percent (26/59) of those randomized to $P P I$ reported $\geq 1$ relevant, acid-related symptom in weeks 9-12 compared with $15 \%$ (9/59; $P<.001)$ in the placebo group. The proportion reporting dyspepsia, heartburn, or acid regurgitation in the PPI group was 13 of 59 (22\%) at week 10, 13 of 59 (22\%) at week 11, and 12 of $58(21 \%)$ at week 12. Corresponding figures in the placebo group were $7 \%$ at week $10(P=.034), 5 \%$ at week $11(P=.013)$, and $2 \%$ at week $12(P=.001)$. Conclusions: PPI therapy for 8 weeks induces acid-related symptoms in healthy volunteers after withdrawal. This study indicates unrecognized aspects of PPI withdrawal and supports the hypothesis that RAHS has clinical implications.

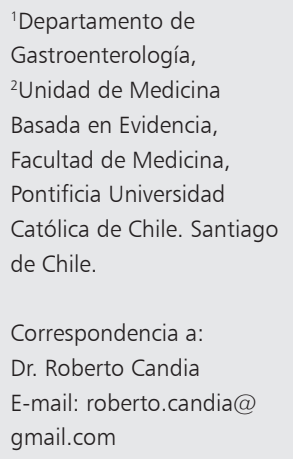

\section{Conclusión de los revisores}

La suspensión de una terapia prolongada con inhibidores de la bomba de protones podría inducir pirosis y sensación de regurgitación en personas previamente asintomáticas.

\section{Pregunta clínica}

¿La suspensión de una terapia crónica con in- hibidores de bomba de protones induce síntomas asociados a reflujo gastroesofágico?

\section{Introducción}

El uso de inhibidores de la bomba de protones (IBP) es frecuente y en constante incremento. Datos epidemiológicos muestran que aproximadamente el 5\% de la población recibe o ha recibido tratamiento a largo plazo con estas drogas ${ }^{1}$, lo que 
está avalado por su alta eficacia en el bloqueo de la secreción ácida gástrica y su excelente perfil de seguridad. Múltiples guías clínicas recomiendan su uso en diferentes escenarios ${ }^{2,3}$ :

- Manejo empírico de dispepsia no ulcerosa.

- Reflujo gastroesofágico (RGE).

- Enfermedad péptica.

- Profilaxis de enfermedad péptica en usuarios crónicos de antinflamatorios no esteroidales (AINEs).

A pesar de lo anterior, se estima que hasta 33\% de los pacientes con uso prolongado no tienen una clara indicación ${ }^{4}$. Algunas de las causas pueden ser deducidas a partir de datos obtenidos en la atención primaria: el retiro del medicamento es difícil de lograr en pacientes con uso crónico, independiente de su indicación, dada la aparición o reaparición de síntomas dispépticos al intentar su suspensión ${ }^{5}$.

Se ha demostrado que el uso de estas drogas por más de 8 semanas induce una hipersecreción de gastrina, mediado en parte por un incremento de la masa de células enterocromafines. La gastrina es el principal estimulador paracrino de la secreción ácida gástrica. Estudios fisiológicos muestran la existencia de un feedback negativo en el control de su secreción: a mayor secreción ácida menor secreción de gastrina y viceversa. Este feedback explicaría los altos niveles plasmáticos de gastrina en los usuarios crónicos de IBP y generaría una hipersecreción ácida de rebote al suspender el medicamento ${ }^{6-8}$. Esta hipersecresión ácida transitoria podría inducir o regenerar los síntomas y, por tanto, producir la necesidad de prolongar el uso de IBP.

\section{Características del estudio}

Tipo de estudio: Estudio clínico randomizado, realizado en un hospital universitario danés entre septiembre de 2007 y marzo de 2008.

Pacientes: Se reclutaron 120 individuos sanos, mayores de 18 años, sin síntomas asociados a secreción ácida (pirosis o regurgitación) y sin uso previo de IBP o bloqueadores del receptor de histamina tipo 2. Se dividieron en 2 grupos de 60 pacientes.

Grupo Intervención: Esomeprazol $40 \mathrm{mg}$ al día por 8 semanas seguido por placebo hasta completar 12 semanas.
Grupo Control: Placebo durante 12 semanas.

Outcomes: Outcome primario: Aparición de síntomas asociados a RGE ácido. Para su evaluación se utilizó una encuesta previamente validada que cuantifica en una escala de 1 a 7 la presencia de dispepsia, regurgitación y pirosis $(1=$ ausencia de síntomas $/ 7$ = síntomas de máxima intensidad). Esta encuesta fue aplicada a través de un cuestionario en línea y los participantes debían contestarla en forma semanal mientras durase el estudio. Se consideró como "síntoma significativo" un puntaje $>2$.

Outcomes secundarios: Niveles plasmáticos de gastrina y cromogranina A, como una estimación indirecta de la secreción ácida gástrica y la masa de células enterocromafines.

\section{Validez (riesgo de sesgo) del estudio}

Ver Tabla 1.

\section{Resultados}

Ver Tabla 2.

\section{Comentarios y aplicación práctica}

\section{Comentarios acerca de la validez \\ (riesgo de sesgo)}

Se trata de un estudio de buen diseño que cumple con la mayoría de los criterios para minimizar el riesgo de sesgo. En relación a la "randomización"

Tabla 1.

\begin{tabular}{|ll|}
\hline 1. Generación adecuada de la secuencia & Sí \\
2. Ocultamiento de la secuencia de & Sí \\
asignación & \\
3. Seguimiento & $98,3 \%$ \\
$\begin{array}{ll}\text { 4. Grupos similares respecto a variables } \\
\text { pronósticas }\end{array}$ & Parcialmente* \\
5. Intención de tratar & No \\
6. Fueron ciegos a la intervención & \\
- Pacientes & Sí \\
- Tratantes & Sí \\
- Recolectores de datos & Sí \\
- Adjudicadores de eventos & Sí \\
- Analistas & No claro \\
\hline
\end{tabular}

* Se pesquisó diferencia en la cantidad de infectados por $\mathrm{H}$. pylori (grupo intervención 2\% vs 13\% grupo control). 
Tabla 2.

\begin{tabular}{|lcclcc|}
\hline Outcome* & \multicolumn{2}{c}{ Efectos absolutos } & \multicolumn{2}{c|}{ Efectos relativos } \\
& $\begin{array}{c}\text { Grupo } \\
\text { Intervención }\end{array}$ & $\begin{array}{c}\text { Grupo } \\
\text { Control }\end{array}$ & Diferencia & RR \\
(IC 95\%)
\end{tabular}

$\mathrm{RR}=$ Riesgo relativo $(\mathrm{RR}), \mathrm{IC}=$ Intervalo de confianza. ${ }^{*}$ No se observaron diferencias en los síntomas entre la semana 0 y 8 , tanto en el puntaje promedio de la encuesta como en la proporción de pacientes con síntomas relevantes.

(distribución aleatoria o al azar, en castellano) y al ocultamiento de la secuencia de asignación, el diseño es apropiado, ya que la secuencia de la distribución aleatoria fue generada por un computador centralizado y la asignación a cada grupo fue realizada a través de códigos en sobres sellados. Otro aspecto relevante del estudio está relacionado con el rol que cumplen los pacientes: ellos son los que aplican la intervención (autoadministración del medicamento) y a la vez son los adjudicadores de outcome (ellos responden semanalmente una encuesta on-line). La utilización de un placebo idéntico, sumado a que los pacientes no estaban informados en relación al momento de la suspensión del IBP, garantizan el "ciego" en personajes importantes para el desarrollo de sesgo.

En relación al análisis de los datos: Sólo se incluyeron en el análisis 118 de los 120 pacientes inicialmente reclutados, por lo tanto, los datos de 2 pacientes están "perdidos". Los pacientes analizados corresponden a los que adhirieron con más de $90 \%$ de la terapia, por lo tanto, estamos frente a un análisis por protocolo. Si bien este tipo de análisis no es el ideal cuando existe pérdida de datos, dado que se altera el balance generado por la "randomización" (en un escenario con pérdida importante de datos el análisis más adecuado es el "por intención de tratar"), en este caso nos parece que el riesgo de sesgo es bajo, por dos razones:

a. Si consideramos que el objetivo del estudio es evaluar un efecto adverso, para poder concluir fehacientemente que la aparición de los síntomas es secundaria al uso previo de IBP los autores deben estar absolutamente seguros que los pacientes consumieron la droga en forma apropiada, por lo tanto, el análisis por protocolo es el indicado en esta situación. b. El riesgo de sesgo está dado por la pérdida de información, independiente del tipo de análisis. Dado que más de $98 \%$ de los pacientes cumplieron con el protocolo, el riesgo de sesgo asociado es menor.

$\mathrm{Al}$ evaluar el balance entre las variables pronósticas basales destaca la diferencia en la tasa de infección por Helicobacter pylori (HP), que es mayor en el grupo control. Existen datos epidemiológicos y fisiopatológicos que muestran que la gastritis crónica por HP con compromiso predominantemente corporal podría disminuir los síntomas asociados a $\mathrm{RGE}^{9,10}$. Esta gastritis disminuye la masa de células parietales, que son las responsables de la secreción ácida; por lo tanto, la mayor tasa de infección en el grupo control podría protegerlos de los síntomas asociados a RGE ácido y generar sesgo. Afortunadamente este riesgo no es categórico, ya que una revisión sistemática no confirma esta asociación $\mathrm{n}^{11} \mathrm{y}$ un estudio posterior, de diseño similar, pero que incluyó sólo pacientes no infectados por HP, muestra resultados en la misma dirección que el estudio analizado ${ }^{12}$.

\section{Comentarios acerca de los resultados}

Este estudio muestra que la suspensión de una terapia crónica con IBP ( 8 semanas) induce síntomas de RGE ácido en pacientes previamente sanos.

- La proporción de pacientes con síntomas de RGE clínicamente relevantes una vez suspendido el IBP fue $20,7 \%$, versus $1,7 \%$ en el grupo que utilizó placebo (semana 12). Si evaluamos la proporción acumulada entre las semanas 8 y 12 de pacientes con síntomas relevantes la diferencia es $44 \%$ vs $15 \%$, respectivamente; es decir, el riesgo de inducir síntomas de RGE al 
suspender IBP versus el no usar terapia prácticamente se triplica.

- Este fenómeno puede ser secundario a hipersecreción ácida de rebote. Entre los outcomes secundarios se incluyó la medición semanal de gastrina y cromogranina A en plasma, ambas como indicadores indirectos de secreción gástrica. Ambos mostraron niveles significativamente más altos en el grupo que recibió IBP y se correlacionaron con la aparición de síntomas. A pesar que estos datos fisiopatológicos apoyan los hallazgos clínicos, este estudio no fue diseñado para demostrar este punto.

- La diferencia en el puntaje promedio de la encuesta aplicada alcanza significación estadística; sin embargo, la diferencia en términos clínicos parece poco relevante: 1,34 vs 1,04 en una escala de 1 a 7 , lo que se contrapone a los hallazgos en el outcome dicotómico (proporción de pacientes con síntomas significativos). Este hallazgo es plausible si el puntaje de los pacientes considerados con o sin síntomas de RGE ácido fue cercano a 2 (umbral para considerar un síntoma como significativo).

- Otro aspecto a considerar es el riesgo de sobrestimar los resultados en términos absolutos: La proporción acumulada de pacientes con síntomas de RGE ácido entre las semanas 8 y 12 en el grupo control es de $15 \%$, la que es llamativamente alta si consideramos que estos eran voluntarios sanos. Una probable explicación es que los pacientes pueden sobrestimar síntomas de menor relevancia al estar obligados a contestar una encuesta semanal y de esa forma exacerbar los hallazgos. Si bien este hecho es cierto, la comparación con placebo permite validar que al menos en términos relativos esta diferencia es real.

- Otro aspecto que apoya la consistencia de los datos es la publicación posterior de un estudio similar que muestra resultados en la misma dirección ${ }^{12}$.

\section{Comentarios acerca de la aplicabilidad}

Los IBP son drogas seguras y ampliamente utilizadas en nuestro medio. En la actualidad son las drogas más efectivas para el manejo de patologías digestivas asociadas a la secreción ácida (RGE, enfermedad péptica, las más frecuentes). La evidencia que avala su seguridad es abundante, incluso en embarazo ${ }^{13,14}$, por lo que los datos ob- tenidos por este estudio son un aporte en relación al perfil de seguridad de estas drogas.

- El estudio está diseñado en voluntarios sanos, lo que permite asegurar que la aparición de síntomas se asocia sólo a la intervención. Si bien se debe asumir que los datos no son del todo aplicables a pacientes con RGE, dado que son poblaciones distintas, si se considera la fisiopatología del síntoma lo más probable es que los hallazgos puedan ser generalizados: en todo paciente los síntomas son secundarios a la presencia de ácido en el esófago, lo que ocurrirá con mayor probabilidad en ambas poblaciones al aumentar la secreción ácida gástrica.

- Los datos proporcionados por este estudio son de alta relevancia en el medio local: La sobreutilización de estas drogas es frecuente y tiene un costo económico asociado tanto para pacientes como para las instituciones prestadoras de salud. Estos resultados nos sugieren que no sólo la indicación inadecuada es responsable de su sobreutilización, si no que la droga en sí, al gatillar síntomas tras su suspensión, obliga a los pacientes a consumirlas crónicamente.

- Este estudio se suma a evidencia reciente que muestra otros efectos no deseados de los IBP, como el riesgo de infecciones gastrointestinales y peritonitis bacteriana espontánea en cirróticos, alteraciones en la absorción de otras drogas $^{15,16}$ y al mayor riesgo de fracturas ${ }^{17,18}$. Si bien la evidencia antes mencionada es controvertida, dado que está originada sólo en estudios observacionales, son aspectos que se deben tener en consideración antes de asumir que estas drogas son inocuas.

En suma, el presente artículo corresponde a un estudio bien diseñado que muestra datos clínicos y fisiopatológicos que sugieren la generación de síntomas asociados a secreción ácida tras la suspensión de drogas diseñadas para lo contrario. $\mathrm{Si}$ bien existe riesgo de sobrestimación de los efectos en términos absolutos, las diferencias en términos relativos son confiables, por lo que esta información se debe considerar al momento de indicar IBP.

\section{Referencias}

1. Forgacs I, Loganayagam A. Over-prescribing proton pump inhibitors. BMJ 2008; 336: 2-3. 
2. Newcastle Guideline Development and Research Unit, NHS National Institute for Clinical Excellence (NICE). Clinical Guideline 17. Dyspepsia-management of dyspepsia in adults in primary care 2004. p. 1-47. Disponible en http://www.nice.org.uk/nicemedia/ live/10950/29460/29460.pdf [Consultado el 17 de febrero de 2012].

3. Talley NJ, Vakil N, Practice Parameters Committee of the American College of Gastroenterology. Guidelines for the management of dyspepsia. Am J Gastroenterol 2005; 100: 2324-37.

4. Raghunath AS, O’Morain C, McLoughlin RC. Review article: the long-term use of proton-pump inhibitors. Aliment Pharmacol Ther 2005; 22 (Suppl 1): 55-63.

5. Martin RM, Lim AG, Kerry SM, Hilton SR. Trends in prescribing $\mathrm{H} 2$-receptor antagonists and proton pump inhibitors in primary care. Aliment Pharmacol Ther 1998; 12: 797-805.

6. Gillen D, Wirz AA, Ardill JE, McColl KE. Rebound hypersecretion after omeprazole and its relation to on-treatment acid suppression and Helicobacter pylori status. Gastroenterology 1999; 116: 239-47.

7. Waldum HL, Arnestad JS, Brenna E, Eide I, Syversen U, Sandvik AK. Marked increase in gastric acid secretory capacity after omeprazole treatment. Gut 1996; 39: 64953.

8. Talley NJ, Meineche-Schmidt V, Pare P, National Institute for Clinical Excellence. Efficacy of omeprazole in functional dyspepsia: double-blind, randomised, placebo-controlled trials (the Bond and Opera studies). Aliment Pharmacol Ther 1998; 12: 1055-65.

9. Labenz J, Blum AL, Bayerdörffer E, Meining A, Stolte M, Börsch G. Curing Helicobacter pylori infection in patients with duodenal ulcer may provoke reflux esophagitis. Gastroenterology 1997; 112 (5): 1442-7.

10. Delaney B, McColl K. Review article: Helicobacter pylori and gastro-oesophageal reflux disease. Aliment Pharmacol Ther 2005; 22 Suppl 1: 32-40.

11. Raghunath AS, Hungin AP, Wooff D, Childs S. Systematic review: the effect of Helicobacter pylori and its eradication on gastro-oesophageal reflux disease in patients with duodenal ulcers or reflux oesophagitis. Aliment Pharmacol Ther 2004; 20 (7): 733-44.

12. Niklasson A, Lindström L, Simrén M, Lindberg G, Björnsson E. Dyspeptic symptom development after discontinuation of a proton pump inhibitor: a doubleblind placebo-controlled trial. Am J Gastroenterol 2010; 105 (7): 1531-7.

13. Gill SK, O’Brien L, Einarson TR, Koren G. The safety of proton pump inhibitors (PPIs) in pregnancy: a metaanalysis. Am J Gastroenterol 2009; 104 (6): 1541-5.

14. Freston JW, Hisada M, Peura DA, Haber MM, Kovacs TO, Atkinson S, et al. The clinical safety of long-term lansoprazole for the maintenance of healed erosive oesophagitis. Aliment Pharmacol Ther 2009; 29 (12): 1249-60.

15. Bajaj J MS, Zadvornova Y, Heuman D, Hafeezullah M, Hoffmann R, Sanyal A, et al. Association of Proton Pump Inhibitor Therapy With Spontaneous Bacterial Peritonitis in Cirrhotic Patients With Ascites. Am J Gastroenterol 104: 1130-4.

16. Lahner E, Annibale B, Delle Fave G. Systematic review: impaired drug absorption related to the co-administration of antisecretory therapy. Aliment Pharmacol Ther 2009; 29 (12): 1219-29.

17. Yu EW, Bauer SR, Bain PA, Bauer DC. Proton pump inhibitors and risk of fractures: a meta-analysis of 11 international studies. Am J Med 2011; 124 (6): 519-26.

18. Eom CS, Park SM, Myung SK, Yun JM, Ahn JS. Use of acid-suppressive drugs and risk of fracture: a metaanalysis of observational studies. Ann Fam Med 201; 9 (3): 257-67. 